\title{
Two-dimensional electron systems in perovskite oxide heterostructures: Role of the polarity-induced substitutional defects
}

\author{
Shih-Chieh Lin $\odot,{ }^{1,2}$ Cheng-Tai Kuo $\odot,, 2,3,{ }^{*}$ Yu-Cheng Shao, ${ }^{4}$ Yi-De Chuang $\odot,{ }^{4}$ Jaap Geessinck $\odot, 5$ Mark Huijben $\odot,{ }^{5}$ \\ Jean-Pascal Rueff, ${ }^{6,7}$ Ismael L. Graff, ${ }^{8}$ Giuseppina Conti, ${ }^{1,2}$ Yingying Peng $\odot,{ }^{9, \dagger}$ Aaron Bostwick, ${ }^{4}$ Eli Rotenberg $\odot,{ }^{4}$ \\ Eric Gullikson, ${ }^{2}$ Slavomír Nemšák $\odot,{ }^{4}$ Arturas Vailionis $\odot,{ }^{10,11}$ Nicolas Gauquelin, ${ }^{5,12}$ Johan Verbeeck, ${ }^{12}$ \\ Giacomo Ghiringhelli, ${ }^{9}$ Claus M. Schneider, ${ }^{1,13}$ and Charles S. Fadley $1,2, \$$ \\ ${ }^{1}$ Department of Physics, University of California Davis, Davis, California 95616, USA \\ ${ }^{2}$ Materials Sciences Division, Lawrence Berkeley National Laboratory, Berkeley, California 94720, USA \\ ${ }^{3}$ Stanford Synchrotron Radiation Lightsource, SLAC National Accelerator Laboratory, Menlo Park, California 94025, USA \\ ${ }^{4}$ Advanced Light Source, Lawrence Berkeley National Laboratory, Berkeley, California 94720, USA \\ ${ }^{5}$ Faculty of Science and Technology and MESA+ Institute for Nanotechnology, University of Twente, Enschede 7500 AE, The Netherlands \\ ${ }^{6}$ Synchrotron SOLEIL, L'Orme des Merisiers, Saint-Aubin-BP48, 91192 Gif-sur-Yvette, France \\ ${ }^{7}$ Sorbonne Université, CNRS,Laboratoire de Chimie Physique-Matière et Rayonnement, 75005 Paris, France \\ ${ }^{8}$ Department of Physics, Federal University of Paraná, Curitiba, Brazil \\ ${ }^{9}$ CNR-SPIN and Dipartimento di Fisica Politecnico di Milano, Piazza Leonardo da Vinci 32, Milano I-20133, Italy \\ ${ }^{10}$ Stanford Nano Shared Facilities, Stanford University, Stanford, California 94305, USA \\ ${ }^{11}$ Department of Physics, Kaunas University of Technology, Studentu Street 50, LT-51368 Kaunas, Lithuania \\ ${ }^{12}$ Electron Microscopy for Materials Science (EMAT), University of Antwerp, Groenenborgerlaan 171, B-2020 Antwerp, Belgium \\ ${ }^{13}$ Peter-Grünberg-Institut PGI-6, Forschungszentrum Jü̈lich, Jü̈lich 52425, Germany
}

(Received 4 March 2020; accepted 29 October 2020; published 19 November 2020)

\begin{abstract}
The discovery of a two-dimensional electron system (2DES) at the interfaces of perovskite oxides such as $\mathrm{LaAlO}_{3}$ and $\mathrm{SrTiO}_{3}$ has motivated enormous efforts in engineering interfacial functionalities with this type of oxide heterostructures. However, the fundamental origins of the 2DES are still not understood, e.g., the microscopic mechanisms of coexisting interface conductivity and magnetism. Here we report a comprehensive spectroscopic investigation on the depth profile of 2DES-relevant Ti $3 d$ interface carriers using depth- and element-specific techniques like standing-wave excited photoemission and resonant inelastic scattering. We found that one type of Ti $3 d$ interface carriers, which give rise to the 2DES are located within three unit cells from the n-type interface in the $\mathrm{SrTiO}_{3}$ layer. Unexpectedly, another type of interface carriers, which are polarity-induced Ti-on-Al antisite defects, reside in the first three unit cells of the opposing $\mathrm{LaAlO}_{3}$ layer $(\sim 10$ $\AA$ ). Our findings provide a microscopic picture of how the localized and mobile Ti $3 d$ interface carriers distribute across the interface and suggest that the 2DES and 2D magnetism at the $\mathrm{LaAlO}_{3} / \mathrm{SrTiO}_{3}$ interface have disparate explanations as originating from different types of interface carriers.
\end{abstract}

DOI: 10.1103/PhysRevMaterials.4.115002

\section{INTRODUCTION}

The discovery of conductivity at the polar-nonpolar interfaces of insulating oxides such as $\mathrm{LaAlO}_{3}$ (LAO) and $\mathrm{SrTiO}_{3}$ (STO) has revealed great potentials for engineering emergent interfacial functionalities absent in their bulk forms [1-5]. The two band insulators LAO and STO have the perovskite structure consisting of a mutual stacking of $(\mathrm{LaO})^{+}$and $\left(\mathrm{AlO}_{2}\right)^{-}$, and $(\mathrm{SrO})^{0}$ and $\left(\mathrm{TiO}_{2}\right)^{0}$ atomic layers, respectively, with their nominal valence values indicated. In the [001] direction, two different interfaces can be formed between the polar LAO and nonpolar STO: $(\mathrm{LaO})^{+} /\left(\mathrm{TiO}_{2}\right)^{0}$ (named $n$-type)

\footnotetext{
*ctkuo@slac.stanford.edu

${ }^{\dagger}$ Present address: International Center for Quantum Materials, School of Physics, Peking University, Beijing 100871, China

${ }^{\ddagger}$ Deceased August 1, 2019.
}

and $(\mathrm{SrO})^{0} /\left(\mathrm{AlO}_{2}\right)^{-}$(named $p$-type) [1-3]. A remarkable feature is that the two-dimensional electron system (2DES) can only form at the $n$-type interface, when the thickness of the top LAO is beyond a critical thickness of three unit cells (uc) [3].

How is this 2DES established in the first place? While there is some consensus that its origin may be associated with the Ti $3 d$ electrons at the interfaces [6], the underlying microscopic mechanisms are far from being understood. A variety of suggestions have been put forward to explain some of the experimental observations, but they usually address only specific aspects and are yet unable to provide a consistent solution to all the puzzles. For example, the mechanism of intrinsic electronic reconstruction [1-3,7] and mechanisms that involve oxygen vacancies [8-11] and interfacial cation intermixing [12-16] are able to interpret the interface conductivity at $n$-type interfaces, but they are difficult to explain the insulating $p$-type interfaces and the interface magnetism. In 
$2014 \mathrm{Yu}$ and Zunger took a very interesting approach and went beyond the view of a mere electronic reconstruction. On the basis of first-principles calculations, they proposed the so-called polarity-induced defect mechanism [17]. It predicts that defects spontaneously form at the LAO surface and/or the LAO/STO interfaces in order to compensate the built-in field induced by the polar discontinuity, and in this way avoid a divergence to the electric potential. Consequently, the formation of 2DES and the appearance of interface magnetism ought to be driven by the polar-field-induced defects, such as the paired antisite and oxygen vacancy defects [17]. Exploring such defects in experiments is a challenging task and calls for sophisticated approaches that are able to extract information specifically from the interface.

$\mathrm{X}$-ray photoemission spectroscopy is a powerful tool for revealing the interface electronic structure and has provided valuable information regarding the electronic reconstruction and quantum confinement effect of these occupied $\mathrm{Ti} 3 d$ states that contribute to 2DES [18-23]. More specifically, resonant x-ray photoemission spectroscopy (RXPS) can enhance the spectral weight of $\mathrm{Ti} 3 d$ states around the Fermi level position and thus has been widely used to study the interface electronic structure of LAO/STO heterostructures [20-23]. By scanning the photon energies across the Ti $2 p$ core level, RXPS can distinguish if the interface carriers are associated with the in-gap (IG) states resulting from oxygen vacancies or the quasiparticle (QP) states from 2DES $[23,24]$. Complementary information about the Ti electronic states can be obtained from resonant inelastic x-ray scattering (RIXS). It probes Ti $3 d$ orbital transitions, namely $d d$ excitations, between occupied and unoccupied states [25-28]. As a drawback, neither conventional photoemission nor RIXS are able to probe the depth profile of the emitted photoelectrons or scattering photons across an interface. Such a profile is strongly needed, however, to evaluate the real space distribution of the Ti $3 d$ carriers in the LAO/STO interface. By contrast, cross-sectional scanning probe microscopies and scanning transmission electron microscopy (STEM) offer high spatial resolution and have been widely used to study the local electronic properties relative to the 2DES of LAO/STO heterostructures [12,29-32], but they cannot resolve the electronic/orbital states. Some advanced STEM studies were able to localize electronic states and hybridization [33] and image orbitals [34] but they are limited in the determination of orbital character due to low- $q$ resolution [33] and subject to instrumental effects and delocalization of the inelastic scattering $[35,36]$.

We have demonstrated in the prior works that standingwave-excited (SW) spectroscopic techniques, such as SWRXPS, SW hard X-ray photoemission spectroscopy (SWHXPS) [37-41], and SW-RIXS [42], are capable of disentangling the physics at buried interfaces in terms of depth profiling the elemental diffusions, polarization-induced voltage drop, and orbital and magnetic excitations. They are thus uniquely suited to elucidate the formation of the 2DES at the LAO/STO interface. In this work, we have, therefore, used a full suite of SW-excited spectroscopic approaches to extract the depth profile of the orbital character of Ti $3 d$ interface electrons across the LAO/STO interfaces and to detail its influence on the interface conductivity and magnetism.
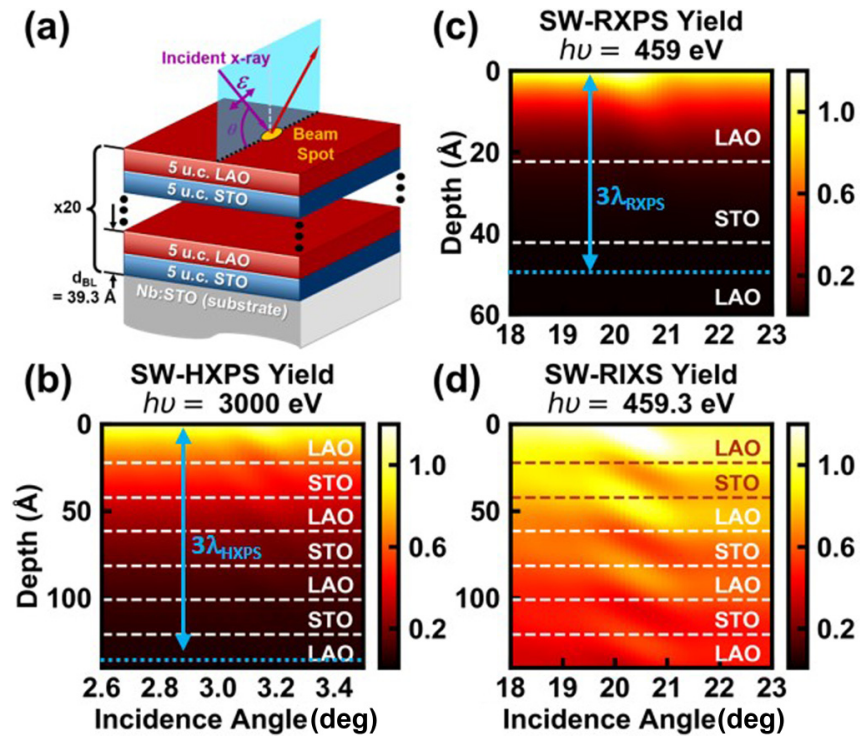

FIG. 1. Depth sensitivity of SW techniques for the LAO/STO superlattice. (a) The superlattice sample, $\left(\mathrm{LAO}_{\mathrm{n}} / \mathrm{STO}_{\mathrm{m}}\right)_{\mathrm{p}}(n=5 \mathrm{uc}$, $m=5 \mathrm{uc}$, and $p=20$ ), was used for the SW measurements by varying the $\mathrm{x}$-ray incidence angles $\theta$ around the Bragg angle $\theta_{\mathrm{B}}$. The photoemission yield distribution of (b) SW-HXPS $(h v=3000 \mathrm{eV})$ and (c) SW-RXPS $(h v=459 \mathrm{eV})$. (d) The RIXS yield distribution of SW-RIXS $(h v=459.3 \mathrm{eV})$. The yield distribution demonstrates the probing depth sensitivity of these techniques. The probing depth for RXPS and HXPS ( $3 \lambda$ ) is $\sim 27$ and $135 \AA$, respectively, as indicated in (b) and (c). The probing depth for RIXS ( $3 \Lambda$ ) is $960 \AA$ [not indicated in (d). According to the probing depths, SW-RXPS is sensitive to the top LAO layer and the first interface, SW-HXPS is sensitive to the top $3 \mathrm{LAO} / \mathrm{STO}$ (STO/LAO) interfaces, and SW-RIXS is sensitive to all $20 \mathrm{LAO} / \mathrm{STO}$ (STO/LAO) interfaces.

\section{RESULTS}

\section{A. Depth sensitivity of $x$-ray standing wave techniques}

Figure 1(a) shows a schematic of SW measurements for this specific sample configuration. The superlattice sample consists of 20 bilayers of 5 uc LAO and 5 uc STO layers on a $\mathrm{TiO}_{2}$-terminated STO (001) substrate. Such limited thicknesses for both layers provide a good basis for visualizing how the 2DES, and possible defects, distribute across the LAO/STO interfaces. Furthermore, the layer thicknesses are chosen such that the superlattice will exhibit metallic behavior with high mobility charge carriers, similar to a single LAO/STO interface system. In a previous study we have shown that adding an additional STO layer on top of the LAO layer triggers the electronic conductivity at a significantly lower LAO film thickness than for the uncapped systems [2], and even a 2 uc STO capping layer is enough to result in very similar metallic behavior as a 10 uc STO capping layer [7], see Supplemental Material Fig. 2 for the typical metallic behavior in our LAO/STO superlattice [43].

The strain condition in our LAO/STO superlattice is equal to the previous studies on LAO/STO interfaces in various sample architectures (single film, bilayer, multilayer or superlattice) on top of single crystalline STO substrates, in which the LAO and STO layers are fully in-plane strained by the underlying STO substrate (3.905 Å) [10]. The STEM 
characterization in the Supplemental Material Fig. 3 [43] shows the controlled epitaxial ordering throughout the full superlattice with a constant in-plane lattice parameter. The out-of-plane lattice parameters of both layers were determined from the peak positions in the x-ray diffraction (XRD) analysis, as shown in the Supplemental Material Fig. 1, to be $\sim 3.73$ $\AA$ and $\sim 3.90 \AA$ for the respective LAO and STO layers. Our superlattice shows the typical shortening of the out-of-plane LAO lattice parameter (instead of $3.791 \AA$ seen in the bulk), while the STO layers remain strain-free [10].

To better demonstrate the difference in the depth sensitivity for these SW techniques, their calculated yield strength distributions are shown in Figs. 1(b)-1(d). Figures 1(b) and 1(c) are the SW-HXPS $(h v=3000 \mathrm{eV})$ and SW-RXPS $(h v=459 \mathrm{eV})$ photoemission yield distributions while Fig. 1(d) is the SW-RIXS $(h v=459.3 \mathrm{eV})$ yield distribution. The probing depth for RXPS, HXPS, and RIXS determined from the yield distributions is $\sim 27,135$, and 960 $\AA$, respectively (see Methods), and the shorter depth for RXPS and HXPS relative to RIXS is due to the shorter escape depth for photoelectrons. Hence, SW-RXPS is sensitive to the top LAO layer and the first interface, SW-HXPS is sensitive to the top three LAO/STO (STO/LAO) interfaces, and SW-RIXS is sensitive to all $20 \mathrm{LAO} / \mathrm{STO}$ (STO/LAO) interfaces. We have combined these different depth sensitivities to obtain a more consistent picture.

Although RIXS has the longest probing depth among these $\mathrm{SW}$ techniques, its signal comes only from the Ti ions in the STO layer, partial information that cannot be used to derive the full sample structure as done with SW photoemission. Such a short-fall can be circumvented by using the core-level rocking curves (RCs) from both SW-HXPS and SW-RXPS to better determine the multilayer structure. We note that the information obtained from HXPS is particularly useful for determining the depth profile of the near-Fermi (NF) peak measured with SW-RXPS and also the $d d$ excitations from SW-RIXS.

\section{B. Standing-wave excited photoemission results}

Figures 2(a) and 2(b) show the experimental core-level spectra and their corresponding RCs (open circles) from representative $\mathrm{La}$ and $\mathrm{Sr}$ core levels at $h v=3000 \mathrm{eV}$ and at $h v=459 \mathrm{eV}$, respectively. The best-fit simulated core-level RCs (curves) are plotted together with the experimental RCs. The best-fit superlattice sample structure was determined by minimizing the total difference between eight experimental (both soft and hard x-rays) RCs and the simulated RCs simultaneously via iteratively adjusting the input structure. The whole fitting results regarding the core-level spectra and their RCs are shown in the Supplemental Material Figs. 6-8 [43]. The determination of best-fit RCs is described in the Methods section. The best-fit results indicate that the superlattice sample has reasonably sharp interface. The averaged interdiffusion between the interfaces is $7 \AA$ with respect to the ideal sample and is in excellent agreement with the STEM results of $1 \sim 3$ uc elemental diffusions (see the Supplemental Material Fig. 3 [43])

Next, we focus on determining the depth profile of the Ti $3 d$ electronic states using SW-RXPS. Using RXPS with (a) $h u=3000 \mathrm{ev}(\mathrm{b})$

(b) $h u=459 \mathrm{eV}$
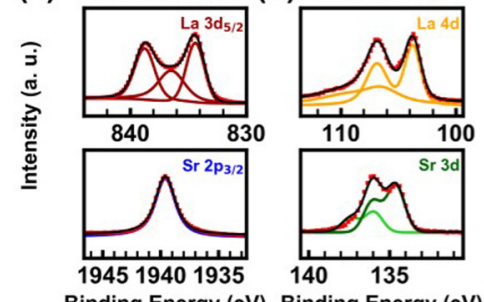

(c) Valence Band $(h v=459 \mathrm{eV})$
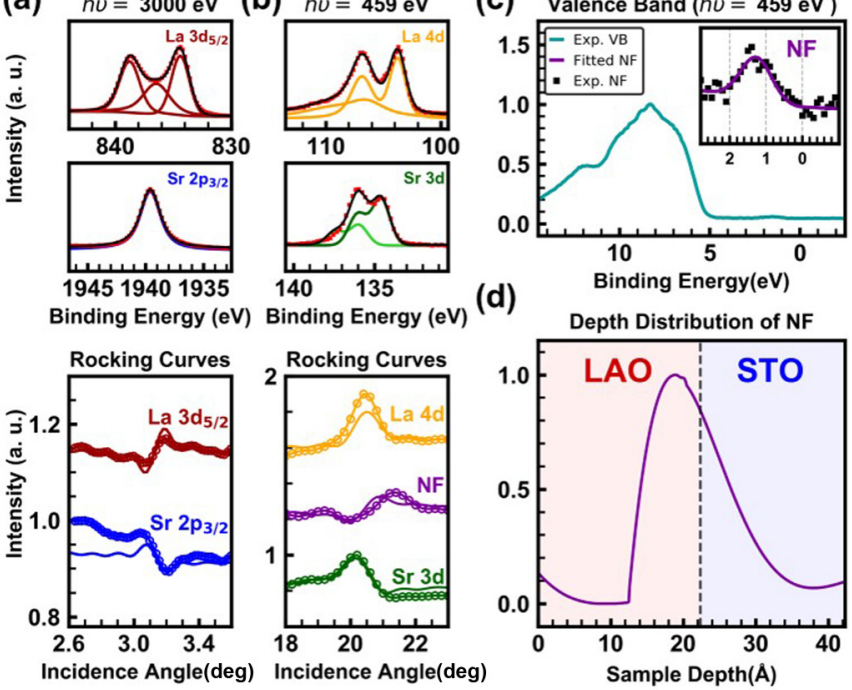

(d)

Depth Distribution of NF

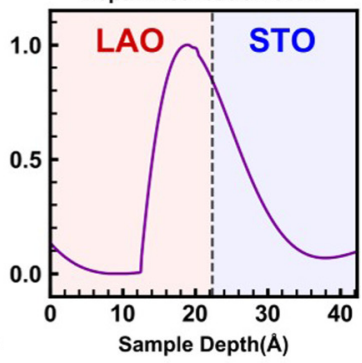

FIG. 2. SW photoemission results and the depth distribution of the near Fermi (NF) peak. (a) SW-HXPS results $(h v=3000 \mathrm{eV})(\mathrm{b})$, (c), (d) SW-RXPS results $(h v=459 \mathrm{eV})$. (a) The top panel is the fitted core-level spectra of $\mathrm{La} 3 d_{5 / 2}$ and $\mathrm{Sr} 2 p_{3 / 2}$ at an off-Bragg angle. The bottom panel shows their experimental (open circle) and best-fit (curve) rocking curves. (b) The top panel is the fitted core-level spectra of $\mathrm{La} 4 d$ and $\mathrm{Sr} 3 d$. The bottom panel shows the experimental and theoretical rocking curves. For the NF peak, its spectrum is shown in c. (c) A valence band spectrum with an inset of the fitted NF peak near the Fermi level. The NF peak is fitted using Voigt function and Fermi-Dirac distribution. (d) The determined depth profile of the NF peak in the top LAO/STO bilayer. The sample depth of zero means the sample surface.

excitation energies near the $\mathrm{TiL}_{3}$ edge, the spectral weight of Ti $3 d$ states around the Fermi level position in the valence band spectrum of LAO/STO heterostructures can be enhanced [20-24]. As mentioned earlier, QP and IG states can be observed around the Fermi level position while excited at the QP and IG resonances. The photon-energy-scanned RXPS map in the Supplemental Material Fig. 5 shows that the chosen energy for SW-RXPS resonance is in the middle of the QP and IG resonances. Due to the limited RXPS energy resolution and the chosen SW resonance, the QP and IG peaks merge into one peak whose centroid shifts to a higher energy. This specific SW-RXPS energy was selected for maximizing the reflectivity/standing wave effect, but apparently it causes the drawback of indistinguishable IG and QP contributions. A detailed discussion can be found in the Supplemental Material Note 3 [43].

Figure 2(c) shows the RXPS valence band spectrum with an inset for a magnified view around the Fermi level where a near Fermi (NF) peak centered at $\sim 0.35 \mathrm{eV}$ is observed. The slightly higher NF peak position with respect to the prior work [20] can be related to the limited energy resolution, and the chosen SW resonance, or from a different level of the impurities in different samples. This NF peak includes both contributions from QS and IG states. The experimental $\mathrm{NF}$ (open circles) and its best-fit (curve) RCs are shown in Fig. 2(b). The contrasting NF RC relative to other core-level RCs indicates that this NF component has its own depth 


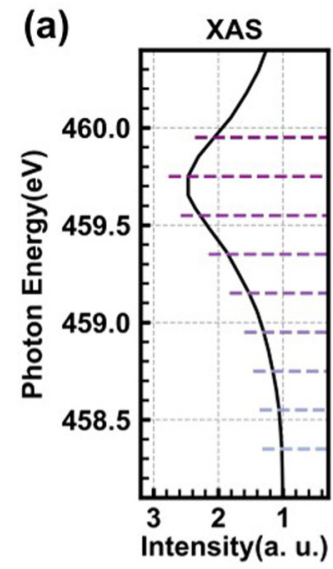

(b)
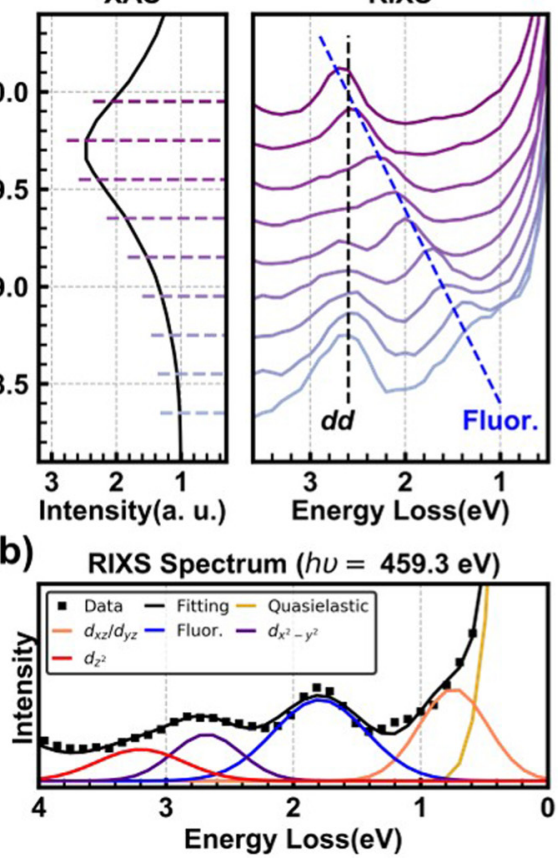

(c) Rocking Curve
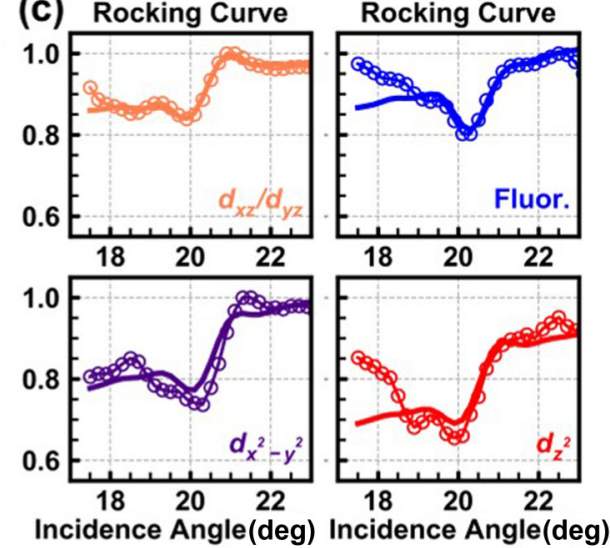

(d)

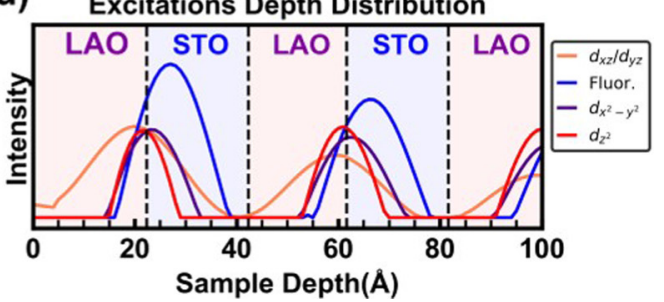

FIG. 3. SW-RIXS result and depth distribution of $d d$ excitations. (a) The excitation-energy-dependent RIXS spectra were collected with excitation energies around the Ti $L_{3}$ XAS edge, ranging from 459.7 to $458.1 \mathrm{eV}$ and marked by color dashed lines in the XAS panel. In the RIXS panel, the black and blue dashed lines are guides to the eyes to show the evolution of the Raman (black) and fluorescence (blue) spectral features, respectively. (b) RIXS spectrum collected using an excitation photon energy of $459.3 \mathrm{eV}$ at an off-Bragg angle for the SW-RIXS measurements. The RIXS spectrum consists of a quasielastic, $d_{x z} / d_{y z}, d_{x^{2}-y^{2}}, d_{z^{2}}$, and fluorescence excitations. (c) Experimental (open circle) and best-fit (curve) rocking curves for $d_{x z} / d_{y z}$, fluorescence, $d_{x^{2}-y^{2}}$, and $d_{z^{2}}$ excitations. The color scheme in this panel adopts that in panel (b). (d) Determined depth profiles of these RIXS excitations in the top five interfaces of LAO/STO heterostructures.

distribution. Since NF is associated with the Ti $3 d$ orbitals, its depth distribution can be related to the $\mathrm{Ti} 3 d$ interface carriers, or the 2DES in the STO layer [20,23,24]. From the depth profile at the first LAO/STO interface [see Fig. 2(d)], our SW-RXPS results suggest that an unexpected source of Ti $3 d$ interface carriers with high concentration is observed in the LAO layer, which will be discussed later.

\section{Standing-wave excited resonant inelastic $x$-ray scattering results}

To gain further insight on the depth distribution of $\mathrm{Ti}$ $3 d$ orbitals and its relationship to the 2DES formation, we have performed SW-RIXS measurements on the very same sample and the results are summarized in Fig. 3. In order to distinguish the Raman-like excitations from the normal fluorescence emission, the excitation-energy-dependent RIXS measurements were performed near the $\mathrm{TiL}_{3}$ edge, see Fig. 3(a) (the wider range energy loss spectrum showing the charge-transfer component can be found in the Supplemental Material Fig. 10 [43]). The $d d$ component shows a constant Raman shift of $\sim 2.6 \mathrm{eV}$, whereas the fluorescence has a linear shift with excitation photon energy. The chosen excitation energy for the SW-RIXS measurements was $1 \mathrm{eV}$ below the $e_{g}$ resonance to better assist probing the $\mathrm{Ti}^{3+}$ ions and separating the $d d$ and fluorescence features. Based on the previous work, we can fit the SW-RIXS spectra with five peaks: quasielastic line, $d_{x z} / d_{y z}(0.8 \mathrm{eV}), d_{x^{2}-y^{2}}(2.7 \mathrm{eV}), d_{z^{2}}$ excitations $(3.2 \mathrm{eV})$, and fluorescence $(\sim 1.8 \mathrm{eV})$, as demonstrated in Fig. 3(b) $[25,27]$.

Their respective experimental RCs (open circles) and bestfit results (curves) are shown in Fig. 3(c). To first order, we can anticipate that the $d d$ excitations are coming from Ti $3 d$ interface carriers with associated structural defects and the fluorescence is from the true 2DES phase. The intensity modulation around the Bragg angle $\left(\sim 20^{\circ}\right)$ for these RCs are evidently different and they also do not fit into the simulated RC from the whole STO layer. Consequently, RIXS excitations must have different spatial distributions. Figure 3(d) shows the determined depth distributions in the top 2.5 LAO/STO bilayers for the RIXS excitations. The depth profile for each excitation in Fig. 3(c) was normalized in a way that its integrated intensity is proportional to the angular-averaged RIXS intensity of the corresponding excitations; therefore, the intensity of the depth profile represents a sort of "concentration" map of excitations in depth.

The SW-RIXS results show that there is a notably large amount of $\mathrm{Ti} 3 d$ interface carriers residing in both the LAO and STO sides of the interface, which is consistent with the SW-RXPS results. These interface carriers reside at the n-type interfaces (LAO_bottom/STO_top) and show no contributions at the p-type interfaces (STO_bottom/LAO_top), agreeing with experimental observations of interface conductivity [1]. In general, a RC with larger (smaller) intensity modulation means a more localized (delocalized) distribution in the depth. The intensity modulations in Fig. 3(c) imply that the fluorescence originate from a wider distribution in depth, while the 


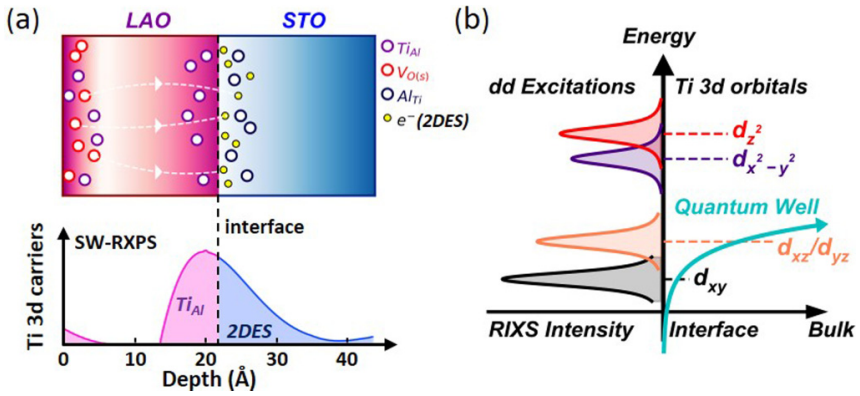

FIG. 4. Formation and subband energy levels of a 2DES at a LAO/STO interface. (a) Illustration of the polarity-induced defect mechanism that leads to the 2DES formation. Paired antisite defects $\left(\mathrm{Ti}_{\mathrm{Al}}\right.$ in the $\mathrm{LAO}$ layer and $\mathrm{Al}_{\mathrm{Ti}}$ in the $\mathrm{STO}$ layer) form in order to alleviate the polarization-induced field across the LAO/STO interfaces. When the LAO layer is below the critical thickness ( $3 \mathrm{uc}$ ), all electrons transferred from $\mathrm{Ti}_{\mathrm{Al}}$ in the LAO layer are trapped by the deep $\mathrm{Al}_{\mathrm{Ti}}$ defects in the STO layer, causing no mobile electron. In contrast, with LAO layer thickness over $3 \mathrm{uc}$, the surface oxygen vacancy defects $\left(\mathrm{V}_{\mathrm{O}(\mathrm{S})}\right)$ start to donate half of the electrons to $\mathrm{Al}_{\mathrm{Ti}}$ defects, and the rest to form the 2DES $\left(e^{-}\right)$in the STO layer. The $\mathrm{Ti}_{\mathrm{Al}}$ defects $\left(\mathrm{Ti}^{3+}\right)$ can be probed by RXPS and RIXS. The lower panel is the SW-RXPS-determined depth profile, showing two main Ti $3 d$ sources. One source in STO is 2DES in the STO and the other is $\mathrm{Ti}_{\mathrm{Al}}$ in the $\mathrm{LAO}$, matching the polarity-induced defect mechanism. (b) The orbital transitions from ground state $\left(d_{x y}\right)$ to final states $\left(d_{x z} / d_{y z}, d_{x^{2}-y^{2}}\right.$, and $\left.d_{z^{2}}\right)$ are responsible for the $d d$ RIXS process. The distributions of these $d d$ excitations are affected by the quantum confinement effect.

$d_{x^{2}-y^{2}}$ excitations originate from narrower distributions. The possible cause of the variations of depth distributions in $d d$ excitations will be discussed later.

\section{DISCUSSION}

The determined depth profile of the NF peak from SWRXPS and the $d d$ excitations from SW-RIXS represent the depth profiles of Ti $3 d$ interface carriers. These results show that, in contrast to the general assumption that these interface carriers are attributed to the mobile 2DES in the STO layer, there are two different sources residing in both LAO and STO. What does this phenomenon stand for? What is the relationship between these interface carriers and the 2DES?

In Fig. 4, we illustrate the polarity-induced defect mechanism for the 2DES formation [17]. The polarity discontinuity across the LAO/STO interfaces triggers the spontaneous formation of multiple kinds of defects. In addition to the surface oxygen vacancy defects $\left(\mathrm{V}_{\mathrm{O}(S)}\right)$, the paired antisite defects such as Ti-on- $\mathrm{Al}\left(\mathrm{Ti}_{\mathrm{Al}}\right)$ and $\mathrm{Al}$-on- $\mathrm{Ti}\left(\mathrm{Al}_{\mathrm{Ti}}\right)$ from the hopping of $\mathrm{Ti}$ atoms near the interface into the $\mathrm{AlO}_{2}$ atomic layers and exchanging site with $\mathrm{Al}$ atoms, can be present in order to alleviate the polarization-induced field across the interfaces. At this n-type interface, when the LAO thickness is below the critical thickness, all electrons are transferred from $\mathrm{Ti}_{\mathrm{Al}}$ to $\mathrm{Al}_{\mathrm{Ti}}$ defects with lowest formation energy to cancel the polar field and subsequently these electrons will be trapped by deep $\mathrm{Al}_{\mathrm{Ti}}$ defects in the STO layer; as a consequence, these electrons become immobile. On the other hand, when the LAO thickness is above the critical thickness, the $\mathrm{V}_{\mathrm{O}(S)}$ defects are responsible for the cancelation of the built-in polar field [17]. Among the electrons ionized from the $\mathrm{V}_{\mathrm{O}(S)}$ defects, half of them are donated to the $\mathrm{Al}_{\mathrm{Ti}}$ defects and the rest form the 2DES in the STO layer. Meanwhile, the interface $\mathrm{Ti}_{\mathrm{Al}}$ defects in the $\mathrm{LAO}$ layer $\left(\mathrm{Ti}^{3+}\right.$ ions on $\mathrm{Al}^{3+}$ sites) donate no electrons. Some of the surface $\mathrm{Ti}_{\mathrm{Al}}$ defects still tend to donate electrons and some remain ionized when the LAO thickness is larger than the critical thickness, but it will gradually be dominated by the $\mathrm{V}_{\mathrm{O}(S)}$ defects with the increased LAO thickness. These polarity-induced substitutional defects in LAO can be probed by RXPS and RIXS, in addition to the 2DES.

The Ti ions in the STO layer can be regarded as the mobile 2DES, residing within the 3 uc STO layer ( $\sim 12 \AA$ in length) near the interface, of which the length scale is fairly consistent with the recent STEM results of $\sim 10 \pm 03 \AA$ [32]. The other $\mathrm{Ti}$ ions located at $\mathrm{LAO}$ layer can be the $\mathrm{Ti}_{\mathrm{Al}}$ defects, residing within $\sim 10 \AA$ LAO layer near the interface. This length scale also perfectly matches the critical thickness of 3 uc predicted by the first-principles calculations [17]. Note that a small concentration of $\mathrm{Ti}_{\mathrm{Al}}$ defects was also found at the LAO surface because some of the $\mathrm{Ti}_{\mathrm{Al}}$ defects are ionized as $\mathrm{Ti}^{3+}$ at the LAO surface; this is also consistent with the polarity-induced defect mechanism [17]. These findings show the strong experimental evidence that substantiates the polarity-induced defect mechanism.

The depth profiles of the NF and $d d$ excitations all show the coexistence of the polarity-induced defects $\left(\mathrm{Ti}_{\mathrm{Al}}\right)$ and 2DES. Additionally, the $\mathrm{Ti}_{\mathrm{Al}}$ defects and 2DES have identical NF peak and energy levels, meaning that they both exhibit orbital reconstructions and quantum confinement effects. This phenomenon implies a strong correlation between these $\mathrm{Ti}_{\mathrm{Al}}$ defects and mobile 2DES. However, the nature of these two Ti interface carriers are still different in terms of the mobility. The polarity-induced defects are localized across the interfaces while the 2DES is mobile. As discovered by the previous HXPS and RIXS results, the derived sheet carrier densities were significantly higher than those obtained by Hall effect measurements, suggesting the coexistence of localized and mobile Ti $3 d$ interface carriers $[18,25]$. In these works, RIXS/HXPS were utilized for the detection of $\mathrm{Ti}^{3+}$ carriers; they found an earlier onset ( 2 uc) of observing Ti $3 d$ interface carriers by RIXS and HXPS compared to that by Hall effect results $(4 \mathrm{uc})$. The quantity of the RIXS/HXPS-determined interface carriers is much higher than that of the Hall-effectdetermined 2DES. Both RIXS and HXPS techniques are not able to tell whether these interface carriers are localized or mobile nor to determine their spatial distribution. Although this phenomenon provides a hint that these RIXS/HXPSdetected carriers are not merely 2DES in the STO layer, the microscopic mechanism is still unclear. Our work provides a reasonable interpretation of this phenomenon. Instead of only observing the 2DES, the RXPS/RIXS experiments observe both the defects and 2DES, because they have $\mathrm{Ti}^{3+}$ characteristics. In other words, when the LAO thickness is less than 3 uc, RIXS/RXPS detect the polarity-induced defects. When the LAO thickness is above the critical thickness, RIXS/RXPS detect both polarity-induced defects and 2DES.

Our findings further provide a clear physical picture of depth distribution of these two coexisting carriers. It is 
noteworthy that, according to the polarity-induced defect mechanism [17], these trapped $\mathrm{Ti}^{3+}$ ions $\left(\mathrm{Ti}_{\mathrm{Al}}\right)$ in LAO layer are responsible for the interface magnetism, while the mobile 2DES in STO layer is responsible for the interface conductivity. The understanding of the interplay of the layers of mobile electrons and trapped ions will be important to enable controlling both interface conductivity and magnetism, which warrants further investigation.

As for the results from SW-RIXS, the variations in the depth distributions of $d d$ excitations in Fig. 3(c) might be seen as insignificant to affect the main messages presented in the previous paragraphs. To give a meaning to the differences in the $\mathrm{RC}$ of the various $d d$ contributions, the subband picture $[19,25,28]$ sketched in Fig. 4(b) can be utilized. Due to the quantum confinement effect, the half-filled $d_{x y}$ orbital is confined at the bottom of a quantum well and has the narrowest depth extension. In contrast, the $d_{x z} / d_{y z}$ orbitals at higher energy are more weakly confined, and this effect is even stronger for the $d_{x^{2}-y^{2}}$ and $d_{z^{2}}$. Finally, the fluorescence signal shows wider distribution with more contributions in STO layer. Indeed, the differences among the $d d$ excitations depth profiles is an intriguing phenomenon, yet difficult to explain at the qualitative level; a more quantitative interpretation will have to include the RIXS cross section and the quantum confinement which goes beyond the scope of present work.

The precision of determining the depth distribution is limited by the SW wavelength, which is $\sim 3.92 \AA$ in this work (for details see the Supplemental Material Note 2 [43]). Therefore, the findings of two kinds of Ti interface carriers residing in the LAO and STO layers are not affected by the energy resolution of RXPS and RIXS. However, the limited energy resolution and the chosen resonance for SW-RXPS leaves a single NF peak without resolving the 2DES and IG states. This means that the depth distribution of 2DES, polarity-induced defects, and oxygen vacancies (IG states) cannot be discriminated. The oxygen vacancies are one type of source to the $\mathrm{Ti}^{3+}$ carriers and they are not necessarily locating merely at the LAO or STO layer. If improving the energy resolution of RXPS, this technique can definitely provide insights to the depth distribution of the oxygen vacancies and the relationship between the oxygen vacancies, polarity-induced defects, and 2DES.

Another future direction of SW spectroscopic studies on the LAO/STO system is to explore the role of oxygen vacancies. Recently, it is found that the LAO/STO interface electronic structure, which includes the QP (associated with 2DES) and IG states (associated with localized electrons), is tunable through the control of the oxygen vacancies $[24,44-$ 46]. SW spectroscopies can show the depth profiles of both the IG and QP states in the oxygen deficient LAO/STO heterostructure, revealing the relationship between oxygen vacancies and $2 \mathrm{DES}$. In addition, the determined 2DES distribution can be related to the width of quantum well that confines the 2DES. Prior work [47] demonstrates the control of the 2DES transport properties upon the electrostatic doping. In this case, the quantum well width across the LAO/STO interfaces is tuned by the variation of a gate voltage. Using SW techniques, one can attain more quantitative understanding of quantum well control, which could be another interesting direction for future studies.
We have demonstrated the coexistence of polarity-induced substitutional defects and 2DES across the polar/non-polar oxide interfaces, implying that the spontaneously formed defects for canceling the interfacial polarization field are the fundamental origins of 2DES formation. Our findings indicate that the well-accepted assumption that the $\mathrm{Ti} 3 d$ interface electrons only exist in the STO side of the interface is an oversimplified picture and should be revised. In addition, the interface conductivity and magnetism at LAO/STO interfaces could originate from different $\mathrm{Ti}$ interface carriers located in the STO and LAO layer, respectively. In a methodological perspective, we have demonstrated the potential of combining SW photoemission and RIXS spectroscopies to obtain the depth distribution of electronic/orbital states at buried interfaces with Angstroms depth precision.

\section{METHODS}

\section{A. Sample synthesis}

The superlattice sample, $\left[\left(\mathrm{LaAlO}_{3}\right)_{\mathrm{n}} /\left(\mathrm{SrTiO}_{3}\right)_{\mathrm{m}}\right]_{\mathrm{p}}$, $\left(\mathrm{LAO}_{\mathrm{n}} / \mathrm{STO}_{\mathrm{m}}\right)_{\mathrm{p}} \quad(n=5$ unit cell (uc), $m=5 \mathrm{uc}$, and $p=20$ ), was deposited by pulsed laser deposition on a $\mathrm{TiO}_{2}$-terminated Nb-doped $\mathrm{SrTiO}_{3}$ (001) substrate. The individual materials were ablated from single-crystalline $\mathrm{LaAlO}_{3}$ and $\mathrm{SrTiO}_{3}$ targets using a $\mathrm{KrF}$ excimer laser operating at $248 \mathrm{~nm}$, while laser fluence and repetition rate were $1.3 \mathrm{~J} / \mathrm{cm}^{2}$ and $1 \mathrm{~Hz}$, respectively. To enable the formation of 2DES interfaces in the LAO/STO multilayers, an oxygen pressure of $2 \times 10^{-3} \mathrm{mbar}$ was applied, while maintaining a growth temperature of $800^{\circ} \mathrm{C}$ $[2,3,7]$. Reflective high-energy electron diffraction indicated layer-by-layer growth mode during the full superlattice growth and was used to enable the precise unit-cell-control of all individual layers.

\section{B. Sample characterization}

XRD analysis confirmed the epitaxial growth of the superlattice as it is fully strained in plane to the STO cubic structure. The highly ordered growth of the superlattice is further demonstrated by the observation of clear Laue fringes between the diffraction peaks (see the Supplemental Material Fig. 1(a) [43]). Analysis of the XRD results showed the coherent growth with a reduced $c$-axis parameter of $3.73 \AA$ for each LAO layer compared to $3.90 \AA$ for each STO layer, which is in good agreement with previous reports [10,48,49]. The low level of surface roughness was confirmed by atomic force microscopy analysis of the surface of LAO/STO superlattice, indicating the presence of smooth terraces separated by clear, single-unit-cell height steps similar to the surface of the initial $\mathrm{TiO}_{2}$-terminated STO (001) substrate (see the Supplemental Material Fig. 1(b) [43]). The structural and chemical quality of the sample have been verified on the local scale STEM, high angle annular dark field (HAADF) imaging ( $Z$ contrast), and electron energy loss spectroscopy (EELS) (See the Supplemental Material Fig. 3 [43]). STEM was performed on a Titan microscope operated at $300 \mathrm{keV}$ with a probe semiconvergence angle of $21 \mathrm{mrad}$; EELS measurements were performed collecting the $\mathrm{Ti}_{.2,3}, \mathrm{Sr} \mathrm{L}_{2,3}$ edge, $\mathrm{O} K$ and La $M_{4,5}$ and $\mathrm{Al} K$ edges simultaneously. The acquisition parameters 
were 0.04 s/pixel, $0.2 \AA$ A $/$ pixel and $0.5 \mathrm{eV} /$ pixel for mapping in the dual EELS mode. Collection semiangles for HAADF imaging and EELS were 41-4 mrad and $94 \mathrm{mrad}$, respectively. STEM results show the superlattice sample has sharp and well-controlled interfaces with high quality that is compatible with the previous works $[12,50]$.

\section{Standing wave excited hard and resonant $x-$ ray photoemission (SW-HXPS and SW-RXPS) measurements}

SW-RXPS measurements were performed at the beamline MAESTRO of Advanced Light Source, Lawrence Berkeley National Laboratory. SW-HXPS measurements were performed at the beamline GALAXIES of SOLEIL synchrotron. The synchrotron radiation light was $p$ polarized. The energy resolution of SW-RXPS is $500 \mathrm{meV}$ and that of SW-HXPS is $440 \mathrm{meV}$. Both measurements were performed at room temperature to prevent the charging effect. The details of GALAXIES endstation can be found elsewhere [51]. In all the RXPS spectra, the contributions of the second-order light were subtracted [24,45] and its details are discussed in the Supplemental Material [43]. The intensities and profiles of the near Fermi level peak were examined to check the $\mathrm{x}$-ray irradiation effect. From the spectra measured before and after a complete set of SW-RXPS measurements ( $\sim 12 \mathrm{~h}$ ), no clear change was observed. Therefore, we conclude that the irradiation effect is below the detection limit presumably due to the low photon flux of $\mathrm{x}$-rays in our experiments.

\section{Standing wave excited resonant inelastic x-ray scattering} (SW-RIXS) measurements

SW-RIXS measurements were performed at the qRIXS endstation at beamline 8.0.1.3 at the Advanced Light Source, Lawrence Berkeley National Laboratory. The combined energy resolution of the SW-RIXS determined from the full width at half maximum of the elastic peak is $360 \mathrm{meV}$. The multilayer sample was cooled down to $\sim 78 \mathrm{~K}$ by liquid $\mathrm{N}_{2}$ during the measurements. The RIXS measurements were probed by $p$-polarized $\mathrm{x}$-ray with a scattering angle of $115^{\circ}$ near the Ti $L_{3}$ edge. The details of qRIXS endstation can be found elsewhere [52].

\section{E. x-ray optics calculations of SW effect in photoemission and RIXS}

The SW is generated by the interference between the incident and reflected waves, and it satisfies this equation $\lambda=$ $2 d_{\mathrm{BL}} \sin \theta_{\mathrm{B}}$, where $\lambda$ is the wavelength of the incident $\mathrm{x}$-rays, $d_{\mathrm{BL}}$ is the thickness of the bilayer, and $\theta_{\mathrm{B}}$ is the Bragg angle. The resulting SW electric-field intensity varies sinusoidally with respect to the sample depth and has a periodicity very close to $d_{\mathrm{BL}}$. Scanning the incidence angle over the Bragg peak moves the SW vertically by half of its wavelength, and this variation provides the phase-sensitive depth resolution for photoemission and RIXS. The vertical movement of the SW through the sample with changing the incidence angle will thus enhance or reduce photoemission/RIXS signals from different depths, generating what are called rocking curves (RC). The introduction to the SW technique can be found in the Supplemental Material.
An X-ray optics computing code (Yang X-ray Optics, YXRO [39]) has been used for generating the yield intensity map and analyzing the experimental $\mathrm{RC}$ data. The probing depth for SW photoemission is defined as $3 \lambda$, where $\lambda$ is the effective inelastic mean free paths. The probing depth for SWRIXS is defined as $3 \Lambda$, where $\Lambda$ is the effective attenuation lengths. The yield intensities are SW intensities multiplied by an attenuation term which takes into account $\lambda_{\text {RXPS }} \backslash \lambda_{\text {HXPS }}$ for the photoelectron peaks or $\Lambda$ for the RIXS excitations peaks. The optical constants used for the simulations with excitation energies near the $\mathrm{TiL}_{3}$ edge were calculated from a Ti XAS spectrum using the Kramers-Kronig relations.

The core-level RCs measured by SW-HXPS and SWRXPS represent the intensities of photoelectrons collected from the elements distributed uniformly in either the LAO or STO layer. Their best-fit RCs are determined by minimizing the errors between the experimental and simulated RCs simultaneously via iteratively adjusting the input structure (e.g. the LAO thickness). The NF RC for SW-RXPS and $d d$ and fluorescence RCs for SW-RIXS do not have uniform distribution and thus have depth profiles with a weighting coefficient for each point at a specific depth. For these kinds of experimental RCs, their best-fit RCs are determined by minimizing the errors between the experimental and simulated RCs simultaneously via optimizing the weighting coefficients (Broyden-Fletcher-Goldfarb-Shanno method). As an example, we choose the NF RC to show the difference between the experimental and simulated RCs using the optimized (bestfit), steplike $\left(d_{\text {delta }}=1 \sim 15 \AA\right)$, and uniform $\left(d_{\text {delta }}=\right.$ whole STO layer) distributions. The optimized distributions show the best results in terms of minimizing the errors. More discussions on determining the depth profiles can be found elsewhere [42].

\section{ACKNOWLEDGMENTS}

We thank G. M. De Luca and L. Braicovich for discussions. Charles S. Fadley was deceased on August 1, 2019. We are grateful for his significant contributions to this work. We thank Advanced Light Source for the access to Beamline 8.0.3 (qRIXS) via Proposal No. 09892 and beamline 7.0.2 (MAESTRO) via Proposal No. RA-00291 that contributed to the results presented here. We thank synchrotron SOLEIL (via Proposal No. 99180118) for the access to Beamline GALAXIES. This work was supported by the U.S. Department of Energy (DOE) under Contract No. DE-AC0205CH11231 (Advanced Light Source), and by DOE Contract No. DE-SC0014697 through the University of California, Davis (S.-C.L., C.-T.K, and C.S.F.), and from the Jülich Research Center, Peter Grünberg Institute, PGI-6. I. L. G. wishes to thank Brazilian scientific agencies CNPQ (Project No. 200789/2017-1) and CAPES (CAPES-PrInt-UFPR) for their financial support. J.V. and N.G. acknowledge funding from the Geconcentreerde Onderzoekacties (GOA) project "Solarpaint" of the University of Antwerp and the European Union's horizon 2020 research and innovation program ESTEEM3 under grant agreement $n^{\circ} 823717$. The Qu-Ant-EM microscope used in this study was partly funded by the Hercules fund from the Flemish Government. 
[1] A. Ohtomo and H. Y. Hwang, A high mobility electron gas at the $\mathrm{LaAlO}_{3} / \mathrm{SrTiO}_{3}$ heterointerface, Nature (London) 427, 423 (2004).

[2] M. Huijben and G. Rijnders. D. H. A. Blank, S. Bals, S. van Aert, J. Verbeeck, G. van Tendeloo, A. Brinkman, and H. Hilgenkamp, Electronically coupled complementary interfaces between perovskite band insulators, Nat. Mater. 5, 556 (2006).

[3] S. Thiel, G. Hammerl, A. Schmehl, C. W. Schneider, and J. Mannhart, Tunable quasi-two-dimensional electron gases in oxide heterostructures, Science 313, 1942 (2006).

[4] A. Brinkman, M. Huijben, M. van Zalk, J. Huijben, U. Zeitler, J. C. Maan, W. G. van Der Wiel, G. Rijnders, D. H. A. Blank, and $\mathrm{H}$. Hilgenkamp, Magnetic effects at the interface between non-magnetic oxides, Nat. Mater. 6, 493 (2007).

[5] H. Y. Hwang, Y. Iwasa, M. Kawasaki, B. Keimer, N. Nagaosa, and Y. Tokura, Emergent phenomena at oxide interfaces, Nat. Mater. 11, 103 (2012).

[6] M. Salluzzo, J. C. Cezar, N. B. Brookes, V. Bisogni, G. M. De Luca, C. Richter, S. Thiel, J. Mannhart, M. Huijben, A. Brinkman, G. Rijnders, and G. Ghiringhelli, Orbital Reconstruction and the Two-Dimensional Electron Gas at the $\mathrm{LaAlO}_{3} / \mathrm{SrTiO}_{3}$ Interface, Phys. Rev. Lett. 102, 166804 (2009).

[7] R. Pentcheva, M. Huijben, K. Otte, W. E. Pickett, J. E. Kleibeuker, J. Hijben, H. Boschker, D. Kockmann, W. Siemons, G. Koster, H. J. W. Zandvliet, G. Rijnders, D. H. A. Blank, H. Hilgenkamp, and A. Brinkman, Parallel Electron-Hole Bilayer Conductivity from Electronic Interface Reconstruction, Phys. Rev. Lett. 104, 166804 (2010).

[8] G. Herranz, M. Basletić, M. Bibes, C. Carrétéro, E. Tafra, E. Jacquet, K. Bouzehouane, C. Deranlot, A. Hamzić, J.-M. Broto, A. Barthélémy, and A. Fert, High Mobility in $\mathrm{LaAlO}_{3} / \mathrm{SrTiO}_{3}$ Heterostructures: Origin, Dimensionality, and Perspectives, Phys. Rev. Lett. 98, 216803 (2007).

[9] C. Cen, S. Thiel, G. Hammerl, G. W. Schneider, K. E. Andersen, C. S. Hellberg, J. Mannhart, and J. Levy, Nanoscale control of an interfacial metal-insulator transition at room temperature, Nat. Mater. 7, 298 (2008).

[10] M. Huijben, A. Brinkman, G. Koster, G. Rijnders, H. Hilgenkamp, and D. H. A. Blank, Structure-property relation of $\mathrm{SrTiO}_{3} / \mathrm{LaAlO}_{3}$ interfaces, Adv. Mater. 21, 1665 (2009).

[11] Z. Zhong, P. X. Xu, and P. J. Kelly, Polarity-induced oxygen vacancies at $\mathrm{LaAlO}_{3} / \mathrm{SrTiO}_{3}$ interfaces,, Phys. Rev. B 82, 165127 (2010).

[12] N. Nakagawa, H. Y. Hwang, and D. A. Muller, Why some interfaces cannot be sharp, Nat. Mater. 5, 204 (2006).

[13] P. R. Willmott, S. A. Pauli, R. Herger, C. M. Schlepütz, D. Martoccia, B. D. Patterson, B. Delley, R. Clarke, D. Kumah, C. Cionca, and Y. Yacoby, Structural Basis For The Conducting Interface Between $\mathrm{LaAlO}_{3}$ and $\mathrm{SrTiO}_{3}$, Phys. Rev. Lett. 99, 155502 (2007).

[14] L. Qiao, T. C. Droubay, T. C. Kaspar, P. V. Sushko, and S. A. Chambers, Cation mixing, band offsets and electric fields at $\mathrm{LaAlO}_{3} / \mathrm{SrTiO}_{3}(001)$ heterojunctions with variable $\mathrm{La}$ :Al atom ratio, Surf. Sci. 605, 1381 (2011).

[15] M. Salluzzo, S. Gariglio, X. Torrelles, Z. Ristic, R. Di Capua, J. Drnec, M. Moretti Sala, G. Ghiringhelli, R. Felici, and N. B. Brookes, Structural and electronic reconstructions at the $\mathrm{LaAlO}_{3} / \mathrm{SrTiO}_{3}$ interface, Adv. Mater. 25, 2333 (2013).

[16] I. Fongkaew, S. Limpijumnong, and W. R. L. Lambrecht, Effects of structural relaxation, and surface termination on two- dimensional electron gas formation at the $\mathrm{LaAlO}_{3} / \mathrm{SrTiO}_{3}(001)$ interface, Phys. Rev. B 92, 155416 (2015).

[17] L. Yu and A. Zunger, A polarity-induced defect mechanism for conductivity and magnetism at polar-nonpolar oxide interfaces, Nat. Commun. 5, 5118 (2014).

[18] M. Sing, G. Berner, K. Go $\beta$, A. Müller, A. Ruff, A. Wetscherek, S. Thiel, J. Mannhart, S. A. Pauli, C. W. Schneider, P. R Willmott, M. Gorgoi, F. Schäfrers, and R. Claessen, Profiling the Interface Electron Gas of $\mathrm{LaAlO}_{3} / \mathrm{SrTiO}_{3}$ Heterostructures with Hard X-Ray Photoelectron Spectroscopy, Phys. Rev. Lett. 102, 176805 (2009).

[19] A. F. Santander-Syro, O. Copie, T. Kondo, F. Fortuna, S. Pailhès, R. Weht, X. G. Qiu, F. Bertran, A. Nicolaou, A. TalebIbrahimi, P. Le Fèvre, G. Herranz, M. Bibes, N. Reyren, Y. Apertet, P. Lecoeur, A. Barthélémy, and M. J. Rozenberg, Twodimensional electron gas with universal subbands at the surface of $\mathrm{SrTiO}_{3}$, Nature (London) 469, 189 (2011).

[20] C. Cancellieri, A. S. Mishchenko, U. Aschauer, A. Filippetti, C. Faber, O. S. Barišić, V. A. Rogalev, T. Schmitt, N. Nagaosa, and V. N. Strocov, Polaronic metal stat at the $\mathrm{LaAlO}_{3} / \mathrm{SrTiO}_{3}$ interface, Nat. Commun. 7, 10386 (2016).

[21] G. Drera, F. Banfi, F. Federici Canova, P. Borghetti, L. Sangaletti, F. Bondino, E. Magnano, J. Huijben, M. Huijben, G. Rijnders, D. H. A. Blank, H. Hilgenkamp, and A. Brinkman, Spectroscopic evidence of in-gap states at the $\mathrm{SrTiO}_{3} / \mathrm{LaAlO}_{3}$ ultrathin interfaces, Appl. Phys. Lett. 98, 052907 (2011).

[22] A. Koitzsch, J. Ocker, M. Knupfer, M. C. Dekker, K. Dörr, B. Büchner, and P. Hoffmann, In-gap electronic structure of $\mathrm{LaAlO}_{3}-\mathrm{SrTiO}_{3}$ heterointerfaces investigated by soft x-ray spectroscopy, Phys. Rev. B 84, 245121 (2011).

[23] G. Berner, M. Sing, H. Fujiwara, A. Yasui, Y. Saitoh, A. Yamasaki, Y. Nishitani, A. Sekiyama, N. Pavlenko, T. Kopp, C. Richter, J. Mannhart, S. Suga, and R. Claessen, Direct kSpace Mapping of the Electronic Structure in an Oxide-Oxide Interface, Phys. Rev. Lett. 110, 247601 (2013).

[24] J. Gabel, M. Zapf, P. Scheiderer, P. Schütz, L. Duty, M Stübinger, C. Schlueter, T.-L. Lee, M. Sing, and R. Claessen, Disentangling specific versus generic doping mechanisms in oxide heterointerfaces, Phys. Rev. B 95, 195109 (2017).

[25] G. Berner, S. Glawion, J. Walde, F. Pfaff, H. Hollmark, L.C. Duda, S. Paetel, C. Richter, J. Mannhart, M. Sing, and R. Claessen, $\mathrm{LaAlO}_{3} / \mathrm{SrTiO}_{3}$ oxide heterostructures studied by resonant inelastic x -ray scattering, Phys. Rev. B 82, 241405(R) (2010).

[26] K.-J. Zhou, M. Radovic, J. Schlappa, V. Strocov, R. Frison, J. Mesot, L. Patthet, and T. Schmitt, Localized and delocalized Ti $3 d$ carriers in $\mathrm{LaAlO}_{3} / \mathrm{SrTiO}_{3}$ superlattice revealed by resonant inelastic x-ray scattering, Phys. Rev. B 83, 201402(R) (2011).

[27] N. C. Plumb, M. Kobayashi, M. Salluzzo, E. Razzoli, C. E. Matt, V. N. Strocov, K. J. Zhou, M. Shi, J. Mesot, T. Schmitt, L. Patthey, and M. Radović, Evolution of the $\mathrm{SrTiO}_{3}$ surface electronic states as a function of $\mathrm{LaAlO}_{3}$ overlayer thickness, Appl. Surf. Sci. 412, 271 (2017).

[28] F. Pfaff, H. Fujiwara, G. Berner, A. Yamasaki, H. Niwa, H. Kiuchi, A. Gloskovskii, W. Drube, J. Gabel, O. Kirilmaz, A. Sekiyama, J. Miyawaki, Y. Harada, S. Suga, M. Sing, and R. Claessen, Raman and fluorescence contributions to the resonant inelastic soft x-ray scattering on $\mathrm{LaAlO}_{3} / \mathrm{SrTiO}_{3}$ heterostructures, Phys. Rev. B 97, 035110 (2018). 
[29] M. Basletic, J.-L. Maurice, C. Carrétéro, G. Herranz, O. copie, M. Bibes, É. Jacquet, K. Bouzehouane, S. Fusil, and A. Barthélémy, Mapping the spatial distribution of charge carriers in $\mathrm{LaAlO}_{3} / \mathrm{SrTiO}_{3}$ heterostructures, Nat. Mater. 7, 621 (2008).

[30] B.-C. Huang, Y.-P. Chiu, P.-C. Huang, W.-C. Wang, V. T. Tra, J.-C. Yang, Q. He, J.-Y. Lin, C.-S. Chang, and Y.-H. Chu, Mapping Band Alignment Across Complex Oxide Heterostructures, Phys. Rev. Lett. 109, 246807 (2012).

[31] P. W. Lee, V. N. Singh, G. Y. Guo, H.-J. Liu, J.-C. Lin, Y.-H. Chu, C. H. Chen, and M.-W. Chu, Hidden lattice instabilities as origin of the conductive interface between insulating $\mathrm{LaAlO}_{3}$ andSrTiO ${ }_{3}$, Nat. Commun. 7, 12773 (2016).

[32] K. Song, S. Ryu, H. Lee, T. R. Paudel, C. T. Koch, B. Park, J. K. Lee, S.-Y. Choi, Y.-M. Kim, J. C. Kim, H. Y. Jeong, M. S. Rzchowski, E. Y. Tsymbal, C.-B. Eom, and S. H. Oh, Direct imaging of the electron liquid at oxide interfaces, Nat. Nano. 13, 198 (2018).

[33] N. Gauquelin, D. G. Hawthorn, G. A. Sawatzky, R. X. Liang, D. A. Bonn, W. N. Hardy, and G. A. Botton, Atomic scale real-space mapping of holes in $\mathrm{YBa}_{2} \mathrm{Cu}_{3} \mathrm{O}_{6+\delta}$, Nat. Commun. 5, 4275 (2014).

[34] S. Löffler, M. Bugnet, N. Gauquelin, S. Lazar, E. Assmann, K. Held, G. A. Botton, and P. Schattschneider, Real-space mapping of electronic orbitals, Ultramicrpscopy 177, 26 (2017).

[35] R. F. Egerton, Scattering delocalization and radiation damage in STEM-EELS, Ultramicroscopy 180, 115 (2017).

[36] J. S. Jeong, M. L. Odlyzko, P. Xu, B. Jalan, and K. A. Mkhoyan, Probing core-electron orbitals by scanning transmission electron microscopy and measuring the delocalization of core-level excitations, Phys. Rev. B 93, 165140 (2016).

[37] A.-X. Gray, C. Papp, B. Balke, S.-H. Yang, M. Huijben, E. Rotenberg, A. Bostwick, S. Ueda, Y. Yamashita, K. Kobayashi, E. M. Gullikson, J. B. Kortright, F. M. F. de Groot, G. Rijnders, D. H. A. Blank, R. Ramesh, and C. S. Fadley, Interface properties of magnetic tunnel junction $\mathrm{La}_{0.7} \mathrm{Sr}_{0.3} \mathrm{MnO}_{3} / \mathrm{SrTiO}_{3}$ superlattices studied by standing-wave excited photoemission spectroscopy, Phys. Rev. B 82, 205116 (2010).

[38] A. M. Kaiser, A. X. Gray, G. Conti, J. Son, A. Greer, A. Perona, A. Rattanachata, A. Y. Saw, A. Bostwick, S. Yang, S.-H. Yang, E. M. Gullikson, J. B. Kortright, S. Stemmer, and C. S. Fadley, Suppression of Near-Fermi Level Electronic States at the Interface in a $\mathrm{LaNiO}_{3} / \mathrm{SrTiO}_{3}$ Superlattice, Phys. Rev. Lett. 107, 116402 (2011).

[39] S.-H. Yang, A. X. Gray, A. M. Kaiser, B. S. Mun, B. C. Sell, J. B. Kortright, and C. S. Fadley, Making use of x-ray optical effects in photoelectron-, Auger electron-, and x-ray emission spectroscopies: Total reflection, standing-wave excitation, and resonant effects, J. Appl. Phys. 113, 073513 (2013).

[40] S.-C. Lin, C.-T. Kuo, R. B. Comes, J. E. Rault, J.-P. Rueff, S. Nemšák, A. Taleb, J. B. Kortright, J. Meyer-Ilse, E. Gullikson, P. V. Sushko, S. R. Spurgeon, M. Gehlmann, M. E. Bowden, L. Plucinski, S. A. Chambers, and C. S. Fadley, Interface properties and built-in potential profile of $\mathrm{LaCrO}_{3} / \mathrm{SrTiO}_{3}$ superlattice determined by standing-wave excited photoemission spectroscopy, Phys. Rev. B 98, 165124 (2018).

[41] C. S. Conlon, G. Conti, S. Nemšák, G. Palsson, R. Moubah, C.-T. Kuo, M. Gehlmann, J. Ciston, J. Rault, J.-P. Rueff, F. Salmassi, W. Stolte, A. Rattanachata, S.-C. Lin, A. Keqi, A. Saw, B. Hjörvarson, and C. S. Fadley, Hard x-ray standingwave photoemission insights into the structure of an epitaxial
$\mathrm{Fe} / \mathrm{MgO}$ multilayer magnetic tunnel junction, J. Appl. Phys. 126, 075305 (2019).

[42] C.-T. Kuo, S.-C. Lin, G. Ghiringhelli, Y. Peng, G. M. De Luca, D. Di Castro, D. Betto, M. Gehlmann, T. Wijnands, M. Huijben, J. Meyer-Ilse, E. Gullikson, J. B. Kortright, A. Vailionis, N. Gauquelin, J. Verbeeck, T. Gerber, G. Balestrino, N. B. Brookes, L. Braicovich, and C. S. Fadley, Depth-resolved resonant inelastic $\mathrm{X}$-ray scattering at a superconductor/halfmetallic-ferromagnet interface through standing wave excitation, Phys. Rev. B 98, 235146 (2018).

[43] See Supplemental Material Material at http://link.aps.org/ supplemental/10.1103/PhysRevMaterials.4.115002 for further results including (1) sample characterization, (2) basic concepts of standing wave techniques, (3) removal of second-order contribution and identification of the NF peak in the RXPS spectrum, (4) fitting of core-level rocking curves, (5) comparison of simulated RCs using various depth distribution, and (6) wide-energy-loss-range RIXS spectra, which includes Refs. [53-56].

[44] S. M Walker, F. Y. Bruno, Z. Wang, A. de la Torre, S. Riccó, A. Tamai, T. K. Kim, M. Hoesch, M. Shi, M. S. Bahramy, P. D. C. King, and F. Baumberger, Carrier-density control of the $\mathrm{SrTiO}_{3}(001)$ surface 2D electron gas studied by ARPES, Adv. Mat. 27, 3894 (2015).

[45] A. Chikina, F. Lechermann, M.-A. Husanu, M. Caputo, C. Cancellieri, X. Wang, T. Schmitt, M. Radovic, and V. N. Strocov, Orbital ordering of the mobile and localized electrons at oxygen-deficient $\mathrm{LaAlO}_{3} / \mathrm{SrTiO}_{3}$ interfaces, ACS NANO 12, 7927 (2018).

[46] V. N. Strocov, A. Chikina, M. Caputo, M.-A. Husanu, F. Bisti, D. Bracher, T. Schmitt, F. Miletto Granozio, C. A. F. Vaz, and F. Lechermann, Electronic phase separation at $\mathrm{LaAlO}_{3} / \mathrm{SrTiO}_{3}$ interfaces tunable by oxygen deficiency, Phys. Rev. Mater. 3, 106001 (2019).

[47] J. Biscaras, S. Hurand, C. Feuillet-Palma, A. Rastagi, R. C. Budhani, N. Reyren, E. Lesne, J. Lesueur, and N. Bergeal, Limit of the electrostatic doping in two-dimensional electron gas of $\mathrm{LaXO}_{3}(\mathrm{X}=\mathrm{Al}, \mathrm{Ti}) / \mathrm{SrTiO}_{3}$, Sci. Rep. 4, 6788 (2015).

[48] M. Huijben, G. Koster, M. K. Kruize, S. Wenderich, J. Verbeeck, S. Bals, E. Slooten, B. Shi, H. J. A. Molegraaf, J. E. Kleibeuker, S. van Aert, J. B. Goedkoop, A. Brinkman, D. H. A. Blank, M. S. Golden, G. van Tendeloo, H. Hilgenkamp, and G. Rijnders, Defect engineering in oxide heterostructures by enhanced oxygen surface exchange, Adv. Funct. Mater. 23, 5240 (2013).

[49] M. Huijben, G. W. J. Hassink, M. P. Stehno, Z. L. Liao, G. Rijnders, A. Brinkman, and G. Koster, Modified spin relaxation mechanism by tunable coupling between interfacial two-dimensional electron gases in correlated oxide heterostructures, Phys. Rev. B 96, 075310 (2017).

[50] M. Huijben, Y. Liu, H. Boschker, V. Lauter, R. Egoavil, J. Verbeeck, S. G. E. te Velthuis, G. Rijnders, and G. Koster, Enhanced local magnetization by interface engineering in perovskite-type correlated oxide heterostructures, Adv. Mater. Interfaces 2, 1400416 (2015).

[51] J.-P. Rueff, J. M. Ablett, D. Céolin, D. Prieur, Th. Moreno, V. Balédent, B. Lassalle-Kaiser, J.-E. Rault, M. Simon, and A. Shukla, The GALAXIES beamline at the SOLEIL synchrotron: Inelastic X-ray scattering and photoelectron spectroscopy in the hard X-ray range, J. Synchrotron Rad. 22, 175 (2015). 
[52] Y.-D. Chuang, X. Feng, A. Cruz, K. Hanzel, A. Brown, A. Spucces, A. Frano, W.-S. Lee, J. Kim, Y.-J. Chen, B. Smith, J. S. Pepper, Y.-C. Shao, S.-W. Huang, L. A. Wray, E. Gullikson, Z.-X. Shen, T. P. Devereaux, A. Tremsin, W. Yang, J. Guo, R. Duarte, and Z. Hussain, Momentum-resolved resonant inelastic soft x-ray scattering (qRIXS) endstation at the ALS, J. Elec. Spec. Relat. Phemon. 146897 (2019), doi: 10.1016/j.elspec.2019.146897.

[53] D. Doennig and R. Pentcheva, Control of orbital reconstruction in $\left(\mathrm{LaAlO}_{3}\right)_{M} /\left(\mathrm{SrTiO}_{3}\right)_{N}(001)$ quantum wells by strain and confinement, Sci. Rep. 5, 7909 (2015).
[54] Z. Huang, X. Renshaw Wang, Z. Q. Liu, W. M. Lü, S. W. Zeng, A. Annadi, W. L. Tan, X. P. Qiu, Y. L. Zhao, M. Salluzzo, J. M. D. Coey, T. Venkatesan, and Ariando, Conducting channel at the $\mathrm{LaAlO}_{3} / \mathrm{SrTiO}_{3}$ interface, Phys. Rev. B 88, 161107(R) (2013).

[55] A. X. Gray, Future directions in standing-wave photoemission, J. Elec. Spec. Relat. Phenom. 195, 399 (2014).

[56] E. Jaeschke, S. Khan, J. Schneider, J. Hastings (Eds.), Synchrotron Light Sources and Free-Electron Lasers (Springer, Cham, 2018), Chap 1. 